\title{
Anthroposophische Konzepte naturwissenschaftlich beleuchtet
}

\section{Arnoud Templeton ${ }^{a}$, Marc Schlaeppi ${ }^{b}$}

a Dr. med., Oberarzt Onkologie/Hämatologie, Kantonsspital St. Gallen

b Dr. med., Oberarzt mbF Onkologie/Hämatologie, Kantonsspital St. Gallen
Korrespondenz:

Dr. Arnoud Templeton und Dr. Marc Schlaeppi Onkologie / Hämatologie Kantonsspital St. Gallen CH-9007 St. Gallen

arnoud.templeton@kssg.ch marc.schlaeppi@kssg.ch
Wer will was Lebendiges erkennen und beschreiben, Sucht erst den Geist herauszutreiben,

Dann hat er die Teile in der Hand, Fehlt leider! nur das geistige Band.

Mephistopheles in Goethes «Faust»

Der Schweizer Arzt Prof. Peter Heusser war von 1995 bis 2008 Dozent für Anthroposophische Medizin an der Universität Bern, wo er bei verschiedenen Forschungsprojekten (medizinische Anthropologie, Ausbildungs-, Grundlagen- und klinische Forschung), massgeblich beteiligt war. Einer seiner Schwerpunkte ist die Ausarbeitung der wissenschaftlichen Grundlagen der Anthroposophischen Medizin.

Seit Juli 2010 ist Heusser Inhaber des Lehrstuhls für Medizintheorie, Integrative und Anthroposophische Medizin an der Universität Witten - Herdecke / Deutschland. Seine Habilitationsschrift ist Anfang 2011 im Schattauer Verlag in Buchform erschienen. prinzipiell auf der denkerischen «Verarbeitung» von unmittelbar oder apparativ gewonnenen Wahrnehmungen. Bei der Erkenntniswissenschaft («Erkennen des Erkennens») oder der Logik liegt insofern ein Sonderfall vor, als Wahrnehmungsorgan und Wahrnehmungsinhalt im Denken zusammenfallen. Dies sei deshalb von Bedeutung, da hier unmittelbar objektive, rein geistige Vorgänge und Inhalte ( $\mathrm{Ge}$ setze») erfasst und erfahrbar werden.

Dass solche Gesetze jedoch keinesfalls auf abstrakte Inhalte beschränkt bleiben, wird in der Folge an Beispielen wie dem «Schneekristallgesetz» aufgezeigt, welches an sich als prinzipiell unabhängige Grösse vorhanden ist (ante rem), welches sich bei gegebenen Bedingungen manifestieren kann (in re) und schliesslich mit Hilfe der denkerisch durchdrungenen Beobachtung erkannt werden kann (post rem). Hieraus ergibt sich im erwähnten Beispiel, dass bestimmte atmosphärische Bedingungen nicht alleinige Ursache

Heusser entwickelt eine Sicht des Menschen, welche physische, lebendige, seelische und geistige, sich bedingende, aber zueinander emergente Ebenen umfasst.

Heusser entwickelt darin methodisch konsequent eine konzeptionelle Integration vorerst unvereinbar erscheinender molekularbiologisch-kausaler Erklärungen der Schulmedizin und immateriell-energetischer Erklärungen der Komplementärmedizin auf einer gemeinsamen Verständigungsgrundlage. Das Werk ist wissenschaftlich ausgerichtet mit einer Fülle von Literaturangaben, welche die anthroposophischen Konzepte von naturwissenschaftlicher Seite erkenntnistheoretisch beleuchten. Als Anleitung für die praktische Anwendung einer anthroposophisch erweiterten Medizin kann dagegen das 2010 veröffentlichte Referenzwerk des Berliner Internisten Matthias Girke empfohlen werden.

Auch wenn die Lektüre dieser Schrift einen aufmerksamen und konzentrierten Leser fordert, wird dieser immer wieder mit erhellenden Erkenntnissen und neuen Offenbarungen belohnt, die ihm das Buch äusserst lesenswert machen.

Heusser geht zunächst auf Grundfragen jeglichen Erkenntnisprozesses und dessen Zusammenhang mit der Wirklichkeit ein. Erkenntnis beruht demnach der Schneekristalle sind, sondern vielmehr die Bedingung darstellen (Bedingungsursache, causa conditionalis), damit sich das durch Beobachtung und Analyse beschreibbare «Schneekristallgesetz» als Wirkursache (causa efficiens) manifestieren kann.

Die Konsequenz, «Gesetze» als eigenständige und von der Materie unabhängige Wirkgrössen zu sehen,

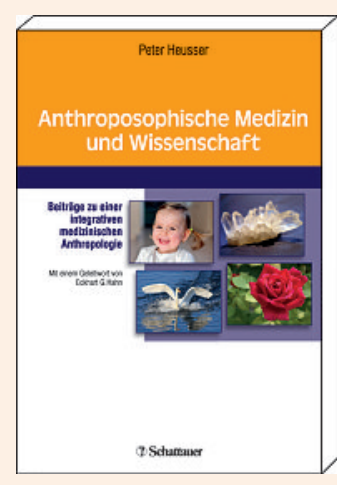

Peter Heusser Anthroposophische Medizin und Wissenschaft Beiträge zu einer integrativen medizinischen Anthropologie. Stuttgart: Schattauer; 2011. 262 Seiten. ISBN 978-3-7945-2807-3 


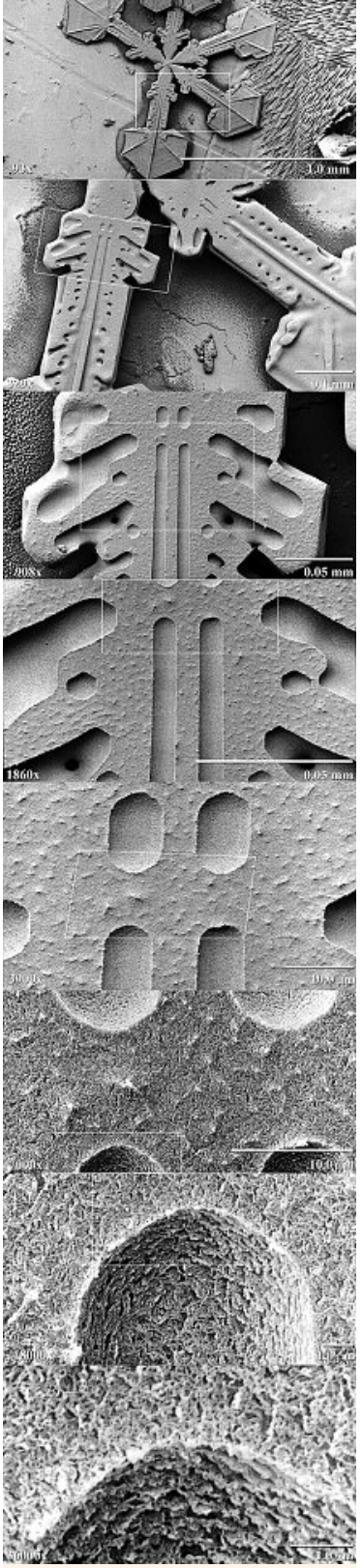

Eiskristall unter dem Elektronenmikroskop: Anhand des «Schneekristallgesetzes» und anderer Beispiele diskutiert Heusser erkenntnistheoretische Fragen. steht zunächst diametral dem reduktionistisch-materialistischen Anspruch entgegen, aus den Eigenschaften der Teile (z.B. Wassermoleküle) Eigenschaften einer höheren Struktur (z. B. Formen der Eiskristalle) voraussagen zu können. Dass nicht einmal die Prädiktion einfachster Kristallstrukturen aus der Kenntnis ihrer chemischen Zusammensetzung möglich sei, wurde entsprechend in einem Nature-Editorial als continuing scandal bezeichnet. Dasselbe gilt für die Nichtvoraussagbarkeit der Tertiärstruktur oder Funktion von Proteinen aus Kenntnis der Primärsequenz der Aminosäuren. Eine Lösung für dieses Problem liegt im Konzept der Emergenz, welche annimmt, dass sich auf einer jeweils höheren, zur niedrigeren emergenten Ebene neue Gesetze manifestieren können, sobald die Bedingungen hierfür vorhanden sind. sität Basel I. P. V. Troxler im Anschluss an Schelling und Hegel gefordert, dass man vom blossen Denken wirkender Gesetze zu einem empirischen Anschauen derselben kommen müsse und für die Wissenschaft des Lebens eine Biosophie, für den multidimensionalen Menschen eine Anthroposophie empfohlen.

Die von R. Steiner begründete anthroposophische Geisteswissenschaft, die sich als Ergänzung zur Anthropologie im herkömmlichen Sinn versteht, hat den Anspruch, durch einen Erkenntnisweg zu eben einer solchen Anschauung zu führen und diese in verschiedenen Praxisfeldern wie der Medizin fruchtbar zu machen.

Im letzten Kapitel gibt Heusser einen Überblick über die aktuelle Situation der anthroposophischen Medizin in Praxis, Lehre und Forschung, wobei neben

\section{Dieses neuste Werk von Peter Heusser kann als rationaler und fundierter Lösungsbeitrag zum Leib-Seele-Problem betrachtet werden.}

In den folgenden Kapiteln entwickelt Heusser mit einer beeindruckenden Detailkenntnis molekularbiologischer Prozesse und der neusten neuropsychologischen Forschung eine Sicht des Menschen, welche physische, lebendige, seelische und geistige, sich bedingende aber zueinander emergente Ebenen umfasst, die jeweils ihren eigenen Gesetzmässigkeiten folgen. Somit wird beispielweise das Gehirn nicht als Ursache, sondern als Bedingung für das Denken verstanden.

Dabei besteht das Problem, dass Naturwissenschaft und Psychologie empirisch lediglich das Bewirkte vor sich haben und allenfalls noch dessen Gesetz in abstrakter Form auffinden können, dass aber mit den Gesetzen assoziierte Wirken selbst empirisch grundsätzlich nicht wahrnehmbar zu sein scheint. Mehrere Denker der europäischen Wissenschaftsgeschichte haben dieses Problem als eine Grenze des Erkennens bezeichnet und eine erkenntnismässige Lösung dafür gesucht. So hatte der Philosoph J. G. Fichte darauf aufmerksam gemacht, dass im Denken nicht nur abstrakter Geist (Ideen, Gesetze), sondern auch wirkender Geist empirisch erlebbar sei, nämlich die vom Ich produzierte Denktätigkeit. Damit beginne im Denken eine höhere, geistige Form der Anschauung.

Novalis nannte diese von Fichte beschriebene höhere Form der Anschauung «Fichtisiren» und empfahl, diese auf «alle Sachen» anzuwenden: «mit dem Ich geht es nur am leichtesten - das ist der Anfang, das Prinzip dieses Gebrauchs». 1828 hatte der Schweizer Arzt und Professor für Philosophie an der Univer- einer Würdigung auch kritisch auf die vielfältigen konzeptionellen und sachlichen Herausforderungen hingewiesen wird.

Dieses neuste Werk von Peter Heusser kann als rationaler und fundierter Lösungsbeitrag zum LeibSeele-Problem betrachtet werden. Es richtet sich in erster Linie an Ärzte und Naturwissenschaftler, kann aber auch uneingeschränkt einer breiten philosophisch-wissenschaftlich bzw. kulturwissenschaftlichen Leserschaft empfohlen werden, die bereit ist, gängige Denkgewohnheiten und, mit dem Augenmerk auf das «geistige Band», den Sinn des «Fichtisirens» zu reflektieren.

\section{Literatur}

- Girke M. Innere Medizin - Grundlagen und Konzepte der Anthroposophischen Medizin. Berlin: Salumed Verlag;2010. Berlin.

- Maddox J. Cristals from first principles. Nature 1988;335:201.

- Fichte J.G. Johann Gottlieb Fichtes Einleitungsvorlesungen in die Wissenschaftslehre, die transcendentale Logik und die Tatsachen des Bewusstseins. Vorgetragen an der Universität zu Berlin in den Jahren 1812 u. 13. Aus dem Nachlass herausgegeben von I.H. Fichte. Bonn: Marcus, 1834.

- Novalis. Novalis Schriften. Die Werke Friedrich von Hardenbergs. Historisch-kritische Ausgabe. Hrsg. von Paul Kluckhon und Richard Samuel. Stuttgart: Kohlhammer; 1960, 2. Auflage.

- Troxler I. P. V. Naturlehre oder Metaphysik. Nach der Erstausgabe 1828 neu hrsg. von Willi Aeppli. Bern: Troxler Verlag; 1944 